\title{
ON THE AVERAGE NUMBER OF REAL ZEROS OF A CLASS OF RANDOM ALGEBRAIC EQUATIONS
}

\author{
N. N. NAYAK and S. BAGH \\ (Received 2 March 1989; revised 17 July 1989)
}

Communicated by Timothy C. Brown

\begin{abstract}
Let $g_{1}, g_{2}, \ldots, g_{n}$ be a sequence of mutually independent, normally distributed, random variables with mathematical expectation zero and variance unity. In this work, we obtain the average number of real zeros of the random algebraic equations $\sum_{k=1}^{n} k^{\sigma} g_{k}(w) t^{k}=C$, where $C$ is a constant independent of $t$ and not necessarily zero. This average is $(1 / \pi)(1+\sqrt{(2 \sigma+1)}) \log n$, when $n$ is large and $\sigma$ is non-negative.
\end{abstract}

1980 Mathematics subject classification (Amer. Math. Soc.) (1985 Revision): 05 A 17, 05 A 19.

\section{Introduction}

Let

$$
P(t)=\sum_{k=1}^{n} k^{\sigma} g_{k}(w) t^{k}
$$

where $g_{1}, g_{2}, \ldots, g_{n}$ is a sequence of mutually independent, normally distributed, random variables with mathematical expectation zero and variance unity. Let $\sigma \geq 0, C$ be a constant independent of $t$ and $N(a, b)$ be the number of real zeros of the algebraic equation $P(t)=C$ in the interval $(a, b)$ with the multiple zeros counted only once. Previously Kac (1943) found that in the case of $C=0$ and $\sigma=0$, the mathematical expectation of the number of real zeros, $E N(-\infty, \infty)$ is asymptotic to $(2 / \pi) \log n$.

(C) 1990 Australian Mathematical Society 0263-6115/90 \$A2.00+0.00 
Again the particular case $\sigma=0$ of the above problem has been considered by Farahmand (1986). In this work we prove the following.

THEOREM. If the coefficients of (1.1) are independent and non-identically distributed random variables, then for any constant $C$, the mathematical expectation of the number of real zeros of the equation $P(t)=C$ satisfies

$$
E N(-\infty, \infty)=\frac{1}{\pi}(1+\sqrt{(2 \sigma+1)}) \log n \text { for large } n .
$$

In order to estimate the average number of real zeros of $P(t)$ in $(-\infty, \infty)$, it is enough to consider the interval $(0, \infty)$ as $E N(-\infty, \infty)=2 E N(0, \infty)$. Now we divide them into two groups,

(i) those lying in the intervals $(0,1-\delta),(1-T / n, 1+T / n)$, and $(1+$ $\delta, \infty)$, and

(ii) those lying in the intervals $(1-\delta, 1-T / n)$ and $(1+T / n, 1+\delta)$, where $\delta=\exp \left(-(\log n)^{1 / 3}\right)$ and $T=(\log n)^{1 / 2}$.

The zeros (i), it so happens, are small in comparison to (ii). So those zeros which make a significant contribution to the final result are of type (ii) and their number is found by the method described below.

\section{Proof of the theorem}

Following the procedure of $\mathrm{Kac}$ (1959), we find

$$
E N(a, b)=\int_{a}^{b} \frac{\left(X_{\sigma} Z_{\sigma}-Y_{\sigma}^{2}\right)^{1 / 2}}{X_{\sigma}} d t
$$

where

$$
X_{\sigma}=\sum_{k=1}^{n} k^{2 \sigma} t^{2 k}, \quad Y_{\sigma}=\sum_{k=1}^{n} k^{2 \sigma+1} t^{2 k-1}
$$

and

$$
Z_{\sigma}=\sum_{k=1}^{n} k^{2 \sigma+2} t^{2 k-2},
$$

provided that $\left(X_{\sigma} Z_{\sigma}-Y_{\sigma}^{2}\right)>0$ in $(a, b)$. We have

$$
X_{\sigma} Z_{\sigma}-Y_{\sigma}^{2}=\frac{1}{2} \sum_{c=1}^{n} \sum_{d=1}^{n}(c d)^{2 \sigma}(d-c)^{2} t^{2(c+d-1)}
$$

From (2.2), we get

$$
\left(\frac{X_{\sigma} Z_{\sigma}-Y_{\sigma}^{2}}{X_{0} Z_{0}-Y_{0}^{2}}\right)<n^{4 \sigma}
$$


Further, for all $t>0$, we have

$$
\frac{X_{\sigma}}{X_{0}}=\frac{\sum_{k=1}^{n} k^{2 \sigma} t^{2 k}}{\sum_{k=1}^{n} t^{2 k}}>\frac{\left(\frac{n}{2}\right)^{2 \sigma}\left(\sum_{[n / 2]}^{n} t^{2 k}\right)}{\left(\sum_{k=1}^{n} t^{2 k}\right)} \geq \frac{(n / 2)^{2 \sigma} t^{n+1}}{t^{n+1}+1}
$$

Again for all $t \geq 1+\delta$, where $\delta=\exp \left(-(\log n)^{1 / 2}\right)$, we have

$$
X_{0}=\sum_{k=1}^{n} t^{2 k}=\frac{t^{2 n+2}}{t^{2}-1}-\frac{t^{2}}{t^{2}-1}
$$

and therefore, for fixed $t$,

$$
\lim _{n \rightarrow \infty}\left(X_{0} / \frac{t^{2 n+2}}{t^{2}-1}\right)=1
$$

So

$$
\begin{aligned}
& X_{0} \sim \frac{t^{2 n+2}}{t^{2}-1} \quad(\text { as } n \rightarrow \infty \text { for fixed } t), \\
& Y_{0}=\sum_{k=1}^{n} k t^{2 k-1}=\frac{n t^{2 n+1}}{\left(t^{2}-1\right)}-\frac{t^{2 n+1}}{\left(t^{2}-1\right)^{2}}+\frac{t}{\left(t^{2}-1\right)^{2}} .
\end{aligned}
$$

So

$$
Y_{0} \sim\left\{\frac{n}{\left(t^{2}-1\right)}-\frac{1}{\left(t^{2}-1\right)^{2}}\right\} t^{2 n+1} \quad(\text { as } n \rightarrow \infty \text { for fixed } t)
$$

and

$$
\begin{aligned}
Z_{0} & =\sum_{k=1}^{n} k^{2} t^{2 k-2} \\
& =\frac{n^{2} t^{2 n}}{\left(t^{2}-1\right)}-\frac{(2 n-1) t^{2 n}}{\left(t^{2}-1\right)^{2}}+\frac{2 t^{2 n}}{\left(t^{2}-1\right)^{3}}-\frac{2 t^{2}}{\left(t^{2}-1\right)^{3}}+\frac{1}{\left(t^{2}-1\right)^{2}}
\end{aligned}
$$

So

$$
Z_{0} \sim\left\{\frac{n^{2}}{\left(t^{2}-1\right)}-\frac{(2 n-1)}{\left(t^{2}-1\right)^{2}}+\frac{2}{\left(t^{2}-1\right)^{3}}\right\} t^{2 n} \quad(\text { as } n \rightarrow \infty \text { for fixed } t)
$$


Now

$$
\begin{aligned}
H(0) & =\left(\frac{X_{0} Z_{0}-Y_{0}^{2}}{X_{0}^{2}}\right)^{1 / 2} \\
& =\left\{\left(\frac{1}{\left(t^{2}-1\right)^{3}}+\frac{1}{\left(t^{2}-1\right)^{4}}\right) t^{4 n+2} / \frac{t^{4 n+4}}{\left(t^{2}-1\right)^{2}}\right\}^{1 / 2} \\
& =\left\{\left(\frac{1}{\left(t^{2}-1\right)}+\frac{1}{\left(t^{2}-1\right)^{2}}\right) \frac{1}{t^{2}}\right\}^{1 / 2} \\
& =\frac{1}{\left(t^{2}-1\right)}
\end{aligned}
$$

If we denote the integrand in (2.1) as $H(\sigma)$, then we get from (2.3) and (2.4) that

$$
\begin{aligned}
H(\sigma) & <4^{\sigma+1} H(0) \quad(\text { for all } t \geq 1+\delta) \\
& =4^{\sigma+1}\left(t^{2}-1\right)^{-1}=\rho(\sigma)\left(t^{2}-1\right)^{-1},
\end{aligned}
$$

where $\rho(\sigma)=4^{\sigma+1}$ and so $\rho(\sigma)$ is a constant depending on $\sigma$ only. Hence it follows from (2.1) that

$$
E N(1+\delta, \infty)=O(\log \delta)
$$

By successive applications of the mean value theorem, we obtain

$$
(k+1)^{2 \sigma+2}-2 k^{2 \sigma+2}+(k-1)^{2 \sigma+2}<(4 \sigma+2)^{2}(k+1)^{2 \sigma} .
$$

Hence

$$
Z_{\sigma}\left(1-t^{t}\right)^{2} t^{2}<X_{\sigma}(4 \sigma+2)^{2} \quad \text { for all } t<1-\delta
$$

that is,

$$
\frac{Z_{\sigma}}{X_{\sigma}}<\frac{(4 \sigma+2)^{2}}{t^{2}\left(1-t^{2}\right)^{2}}
$$

Therefore it follows from (2.1) that

$$
E N(0,1-\delta)=O(\log \delta) \text {. }
$$

Again, clearly $H(\sigma)<n$ for $1-T / n \leq t \leq 1+T / n$, where $T=(\log n)^{1 / 2}$. So from $(2.1)$, we get

$$
E N(1-T / n, 1+T / n)=O\left((\log n)^{1 / 2}\right) .
$$

3. The range $(1+T / n, 1+\delta)$

For $t$ in the range $(1+T / n, 1+\delta)$ we follow the procedure of Das (1969). We replace the random variables $g_{k}$ by a set of independent and continuous 
functions $g_{k}(\tau) \quad(0 \leq \tau \leq 1)$ and denote the right hand side of equation (1.1) by $g(t, \tau)$. Put

$$
h(\tau)=h(\tau, \alpha, \beta)= \begin{cases}1 & \text { if } g(\alpha, \tau) \cdot g(\beta, \tau)<0, \\ \frac{1}{2} & \text { if } g(\alpha, \tau) \cdot g(\beta, \tau)=0, \\ 0 & \text { if } g(\alpha, \tau) \cdot g(\beta, \tau)>0,\end{cases}
$$

and define $N(\tau)=N(\tau ; \alpha, \beta)$ to be the number of zeros of $g(t, \tau)$ in the interval $\alpha \leq t \leq \beta$, reckoned according to their multiplicities except for zeros at $\alpha$ and $\beta$ which are reckoned according to half their multiplicities. Let $\gamma=n(\beta-\alpha)$. Then we have

LEMMA 1.

$$
0 \leq \int_{0}^{1}\{N(\tau)-h(\tau)\} d \tau \leq K \gamma^{2}\left\{\log \frac{1}{\gamma}\right\}^{1 / 2},
$$

where $K$ is an absolute constant.

Proof. The proof follows from Lemmas 2, 3 and 4 of Das (1969), by putting

$$
\boldsymbol{\Phi}^{2}(t)=\sum_{\nu=1}^{n}\left\{\nu^{\sigma+1}(\nu-1) \cdots(\nu-k-1) t^{\nu-k}\right\}^{2}
$$

and

$$
P^{2}(t)=\sum_{\nu=1}^{n} \nu^{2 \sigma} t^{2 \nu}
$$

Let $\Delta=\exp \left\{(\log n)^{1 / 3}\right\} / n^{2}$ and define $q_{0}, q_{1}, \alpha_{q}, \beta_{q}$ by

$$
\begin{aligned}
& q_{0} \Delta<\frac{(\log n)^{1 / 2}}{n}<\left(q_{0}+1\right) \Delta, \\
& q_{1} \Delta<\exp \left\{-(\log n)^{1 / 3}\right\}<\left(q_{1}+1\right) \Delta
\end{aligned}
$$

and

$$
\alpha_{q}=1+q \Delta, \quad \beta_{q}=1+(q+1) \Delta .
$$

Let $N_{q}(\tau), h_{q}(\tau), \gamma_{q}$ be the functions $N(\tau), h(\tau), \gamma$ for the interval $\left(\alpha_{q}, \beta_{q}\right)$ so that

$$
\gamma_{q}=n\left(\beta_{q}-\alpha_{q}\right)=\frac{\exp \left\{(\log n)^{1 / 3}\right\}}{n} .
$$

Now by Lemma 1 , we obtain

$$
\int_{0}^{1}\left\{\sum_{q=q_{0}}^{q_{1}}\left(N_{q}(\tau)-h_{q}(\tau)\right)\right\} d \tau=O\left((\log n)^{1 / 2}\right) .
$$


So

$$
\begin{aligned}
\int_{0}^{1} N\left(\tau ; 1+\frac{T}{n}, 1+\delta\right) d \tau & =\int_{0}^{1}\left(\sum_{q=q_{0}}^{q_{1}} N_{q}(\tau)\right) d \tau \\
& =\left(\sum_{q=q_{0}}^{q_{1}} \int_{0}^{1} h_{q}(\tau) d \tau\right)+O\left((\log n)^{1 / 2}\right)
\end{aligned}
$$

Now following the procedure of Das (1969) and using the estimate

$$
X_{\sigma}=\sum_{k=1}^{n} k^{2 \sigma} t^{2 k} \sim \frac{n^{2 \sigma} t^{2 n+2}}{t^{2}-1}
$$

we obtain

$$
\int_{0}^{1} h_{q}(\tau) d \tau=\frac{1}{\pi} \sin ^{-1}\left(\eta_{q}\right)
$$

where

$$
\eta_{q}^{2}=1-\frac{\left(\sum_{k=1}^{n} k^{2 \sigma} \alpha_{q}^{k} \beta_{q}^{k}\right)^{2}}{\left(\sum_{k=1}^{n} k^{2 \sigma} \alpha_{q}^{2 k}\right)\left(\sum_{k=1}^{n} k^{2 \sigma} \beta_{q}^{2 k}\right)} \sim\left(\frac{\beta_{q}-\alpha_{q}}{\beta_{q} \alpha_{q}-1}\right)^{2} .
$$

Therefore

$$
\int_{0}^{1} h_{q}(\tau) d \tau=\frac{1}{2 \pi q}+o(\Delta)
$$

Hence

$$
\begin{aligned}
E N\left(1+\frac{T}{n}, 1+\delta\right) & =\int_{0}^{1} N\left(\tau ; 1+\frac{T}{n}, 1+\delta\right) d \tau \\
& =\frac{1}{2 \pi} \log n+O(\log n)^{1 / 2}
\end{aligned}
$$

\section{The range $(1-\delta, 1-T / n)$}

For $t$ in the range $(1-\delta, 1-T / n)$, we have to follow the methods given by Logan and Shepp (1968). The expected number of zeros of $P(t)=C$ in the interval $(a, b)$ is given by the Kac-Rice formula:

$$
E N(a, b)=\int_{a}^{b} f_{n}(t) d t
$$

where

$$
f_{n}(t)=\int_{-\infty}^{\infty}|y| p(0, y) d y
$$


and $p(x, y)$ is the probability density for

$$
P(t)=\left(\sum_{k=1}^{n} k^{\sigma} g_{k} t^{k}\right)-C=x
$$

and

$$
P^{\prime}(t)=\sum_{k=1}^{n} k^{\sigma+1} g_{k} t^{k-1}=y .
$$

The distribution of each coefficient $g_{k}$ has the characteristic function $\exp \left(-Z^{2} / 2\right)$ for $-\infty<Z<\infty$.

If $\Phi(z, w)$ is the joint characteristic function of $x$ and $y$, then

$$
\begin{aligned}
\Phi(z, w) & =E\left(\exp \left(i P(t) z+i P^{\prime}(t) w\right)\right) \\
& =\exp \left\{-\frac{1}{2} \sum_{k=1}^{n}\left(a_{k} z+b_{k} w\right)^{2}-i z C\right\}
\end{aligned}
$$

where $a_{k}=k^{\sigma} t^{k}$ and $b_{k}=k^{\sigma+1} t^{k-1}$. Thus the probability density $p(x, y)$ for $P(t)=x$ and $P^{\prime}(t)=y$ is given (Cramer (1954), page 101) by the Fourier inversion formula

$$
\begin{aligned}
p(x, y)= & \frac{1}{(2 \pi)^{2}} \int_{-\infty}^{\infty} \int_{-\infty}^{\infty} \exp (-i x z-i y w) \Phi(z, w) d z d w \\
= & \frac{1}{(2 \pi)^{2}} \int_{-\infty}^{\infty} \int_{-\infty}^{\infty} \exp (-i(x z+y w)) \\
& \times \exp \left(-\frac{1}{2} \sum_{k=1}^{n}\left(a_{k} z+b_{k} w\right)^{2}-i z C\right) d z d w
\end{aligned}
$$

Now

$$
\begin{aligned}
p(0, y)= & \frac{1}{(2 \pi)^{2}} \int_{-\infty}^{\infty} d w \int_{-\infty}^{\infty} \exp (-i y w) \\
& \times \exp \left(-\frac{1}{2} \sum_{k=1}^{n}\left(a_{k} z+b_{k} w\right)^{2}-i z C\right) d z .
\end{aligned}
$$

Then for $\varepsilon>0$, we have

$$
\begin{aligned}
\int_{-\infty}^{\infty}|y| e^{-\varepsilon|y|} p(0, y) d y & \\
=R l \int_{-\infty}^{\infty}|y| e^{-\varepsilon|y|} & {\left[\frac{1}{(2 \pi)^{2}} \int_{-\infty}^{\infty} d w \int_{-\infty}^{\infty} \exp (-i y w)\right.} \\
& \left.\quad \times \exp \left(-\frac{1}{2} \sum_{k=1}^{n}\left(a_{k} z+b_{k} w\right)^{2}-i z C\right) d z\right] d y
\end{aligned}
$$


Now

$$
R l \int_{-\infty}^{\infty}|y| e^{-\varepsilon|y|} \exp (-i y w) d y=\frac{2\left(\varepsilon^{2}-w^{2}\right)}{\left(\varepsilon^{2}+w^{2}\right)^{2}}
$$

so (4.6) becomes

$$
\begin{aligned}
& \text { (4.7) } \int_{-\infty}^{\infty}|y| e^{-\varepsilon|y|} p(0, y) d y \\
& =\frac{1}{\pi^{2}} \int_{0}^{\infty} \frac{\left(\varepsilon^{2}-w^{2}\right)}{\left(\varepsilon^{2}+w^{2}\right)^{2}} d w \int_{-\infty}^{\infty} \exp \left(-\frac{1}{2} \sum_{k=1}^{n}\left(a_{k} z+b_{k} w\right)^{2}\right) d z .
\end{aligned}
$$

Let $A$ and $B$ be arbitrary nonzero constants. If we take $a_{k}$ and $b_{k}$ $(k=1,2, \ldots, n)$ to be any constants independent of $t$, then the probability density $p(x, y)$ corresponding to $x=\sum a_{k} g_{k}=A \bar{x}$ and $y=\sum b_{k} g_{k}=B \bar{y}$ is zero. Further, given $A$ and $B$, the constants $a_{k}$ and $b_{k}$ can be so chosen that the $\bar{x}$ and $\bar{y}$ are normally distributed with variance unity. Thus (4.7) becomes

$$
0=\frac{1}{\pi^{2}} \int_{0}^{\infty} \frac{\left(\varepsilon^{2}-w^{2}\right)}{\left(\varepsilon^{2}+w^{2}\right)^{2}} d w \int_{-\infty}^{\infty} \exp \left(-\frac{1}{2}(A z+B w)^{2}\right) d z
$$

Subtracting (4.8) from (4.7), we obtain

$$
\begin{aligned}
\int_{-\infty}^{\infty}|y| e^{-\varepsilon|y|} p(0, y) d y=\frac{1}{\pi^{2}} \int_{0}^{\infty} \frac{\left(\varepsilon^{2}-w^{2}\right)}{\left(\varepsilon^{2}+w^{2}\right)^{2}} d w & \\
\times\left[\int_{-\infty}^{\infty} \exp \left(-\frac{1}{2} \sum_{k=1}^{n}\left(a_{k} z+b_{k} w\right)^{2}\right)\right. & \left.-\exp \left(-\frac{1}{2}(A z+B w)^{2}\right) d z\right] .
\end{aligned}
$$

Let $\varepsilon \rightarrow 0$, so that we have

$$
\begin{aligned}
\int_{-\infty}^{\infty}|y| p(0, y) & d y=\frac{1}{\pi^{2}} \int_{-\infty}^{\infty} \frac{d w}{w^{2}} \\
\times\left[\int_{0}^{\infty} \exp \left(-\frac{1}{2}(A z+b w)^{2}\right)\right. & \left.-\exp \left(-\frac{1}{2} \sum_{k=1}^{n}\left(a_{k} z+b_{k} w\right)^{2}\right) d z\right],
\end{aligned}
$$

where we put $z=u w$ and use Frullani's theorem (Williamson (1955), page $155)$ to integrate with respect to $w$. So

$$
\int_{-\infty}^{\infty}|y| p(0, y) d y=\frac{1}{2 \pi^{2}} \int_{-\infty}^{\infty} \log \left\{\frac{\sum_{k=1}^{n}\left(a_{k} u-b_{k}\right)^{2}}{(A u-B)^{2}}\right\} d u
$$


Choose $A$ and $B$ so that $A^{2}=\sum_{k=1}^{n} a_{k}^{2}$ and $A B=\sum_{k=1}^{n} a_{k} b_{k}$. Then after simplification (4.9) becomes

$$
\int_{-\infty}^{\infty}|y| p(0, y) d y=\frac{1}{2 \pi^{2} t} \int_{-\infty}^{\infty} \log \frac{\left\{\sum_{k=1}^{n}(u-k)^{2} \Phi_{k}\left(t^{2}\right)\right\}}{\left\{u-\Phi\left(t^{2}\right)\right\}^{2}} d u
$$

where

$$
\Phi_{k}(t)=\frac{k^{2 \sigma} t^{k}}{\sum_{1}^{n} j^{2 \sigma} t^{j}} \quad \text { and } \quad \Phi(t)=\sum_{1}^{n} k \Phi_{k}(t) .
$$

Put $a=1-\delta, b=1-T / n, t=\exp (-\tau / 2 n)$ and $u=n v / \tau$. So we have

$$
\begin{aligned}
E N(1-\delta, 1-T / n) & =\int_{1-\delta}^{1-T / n} f_{n}(t) d t \\
& =\frac{1}{4 \pi^{2}} \int_{T_{0}}^{n \delta_{0}} \frac{d \tau}{\tau} \int_{-\infty}^{\infty} \log \left(\frac{v^{2}-2 j(\tau) v+h(\tau)}{v^{2}-2 j(\tau) v+j^{2}(\tau)}\right) d v,
\end{aligned}
$$

where $T_{0}=-2 n \log (1-T / n)$ and $\delta_{0}=-2 \log (1-\delta)$,

$$
j(\tau)=\frac{\tau}{n} \frac{\left(\sum_{k=1}^{n} k^{2 \sigma+1} \exp \left(-\frac{k \tau}{n}\right)\right)}{\left(\sum_{k=1}^{n} k^{2 \sigma} \exp \left(-\frac{k \tau}{n}\right)\right)}
$$

and

$$
h(t)=\frac{\tau^{2}}{n^{2}} \frac{\left(\sum_{k=1}^{n} k^{2 \sigma+2} \exp \left(-\frac{k \tau}{n}\right)\right)}{\left(\sum_{k=1}^{n} k^{2 \sigma} \exp \left(-\frac{k \tau}{n}\right)\right)} .
$$

Let $\tau=n \eta$ and we have

$$
n^{\nu} e^{-(n+1) \eta}+\left(1-e^{-\eta}\right)\left(\sum_{k=1}^{n} k^{\nu} e^{-k \eta}\right)=\sum_{k=1}^{n} e^{-k \eta}\left\{k^{\nu}-(k-1)^{\nu}\right\}
$$

By the mean value theorem, the last expression is equal to

$$
\nu \sum_{k=1}^{n} e^{-k \eta}(k-\theta)^{\nu-1}
$$

for some $\theta$ with $0<\theta<1$. Again

$$
\begin{aligned}
\sum_{k=1}^{n} e^{-k \eta} k^{\nu-1} & \geq\left(\frac{n-1}{2}\right)^{\nu-1} \sum_{[n / 2]}^{n} e^{-k \eta} \\
& \geq\left(\frac{n-1}{2}\right)^{\nu-1} \frac{\left(e^{-n \eta / 2}-e^{-n \eta}\right)}{\left(1-e^{-\eta}\right)} \\
& >\left(\frac{C_{0}}{n \eta}\right) n^{\nu} e^{-n \eta / 2} \text { for } \frac{1}{2 n}(\log n)^{1 / 2}<\eta<\delta_{0},
\end{aligned}
$$


where $C_{0}$ is a constant depending on $\nu$ only. Thus

$$
\frac{n^{\nu} e^{-(n+1) \eta}}{\sum_{k=1}^{n} e^{-k \eta} k^{\nu-1}}<\frac{n \eta}{C_{0}} e^{-((n+2) \eta) / 2} \rightarrow 0 \quad \text { as } n \rightarrow \infty .
$$

Again

$$
1-e^{-\eta}=\eta+O\left(\eta^{2}\right) \quad \text { as } n \rightarrow 0 .
$$

It can be seen that $\sum_{k=1}^{n} e^{-k \eta}(k-\theta)^{\nu-1}$ lies between $e^{-\eta} \sum_{k=1}^{n} e^{-k \eta} k^{\nu-1}$ and $\sum_{k=1}^{n} e^{-k \eta} k^{\nu-1}$, which are nearly equal. Now dividing equality (4.11) by $\sum_{k=1}^{n} k^{\nu-1} e^{-k \eta}$ and making use of the above relations, we get

$$
\begin{aligned}
\frac{\eta\left(\sum_{k=1}^{n} k^{\nu} e^{-k \eta}\right)}{\left(\sum_{k=1}^{n} k^{\nu-1} e^{-k \eta}\right)} & =\nu+O\left(n \eta e^{-(n \eta) / 2}\right) \\
& =\nu+O\left(\tau e^{-\tau / 2}\right) \quad(\tau \rightarrow \infty) .
\end{aligned}
$$

Putting $\nu=2 \sigma+1$, we find

$$
j(t)=(2 \sigma+1)+O\left(\tau e^{-\tau / 2}\right) .
$$

Similarly we estimate

$$
h(t)=(2 \sigma+2)(2 \sigma+1)+O\left(\tau e^{-\tau / 2}\right) \quad \text { for } \frac{1}{2}(\log n)^{1 / 2}<\tau<n \delta_{0} .
$$

Now $T_{0}=-2 n \log \left(1-\frac{T}{n}\right) \sim \frac{1}{2}(\log n)^{1 / 2}$, so $j(t) \sim(2 \sigma+1)$ and $h(t) \sim$ $(2 \sigma+2)(2 \sigma+1)$. Now

$$
\begin{aligned}
\int_{|v|>M} & \log \left\{\frac{\left(v^{2}-2 j v+h\right)}{\left(v^{2}-2 j v+j^{2}\right)}\right\} d v \\
= & M \log \left\{\frac{\left(M^{2}-2 j M+h\right)}{\left(M^{2}-2 j M+j^{2}\right)} \frac{\left(M^{2}+2 j M+h\right)}{\left(M^{2}+2 j M+j^{2}\right)}\right\} \\
& +j \log \left\{\frac{\left(M^{2}-2 j M+j^{2}\right)}{\left(M^{2}-2 j M+h\right)} \frac{\left(M^{2}+2 j M+h\right)}{\left(M^{2}+2 M+j^{2}\right)}\right\} \\
& +4(h-j)^{2} \int_{M}^{\infty}\left\{\frac{\left(v^{2}+j\right)}{\left(v^{2}+h\right)^{2}-4 j^{2} v^{2}}\right\} d v .
\end{aligned}
$$

Now

$$
\text { (4.13) } \frac{1}{\pi^{2}} \int_{T_{0}}^{n \delta_{0}} \frac{d \tau}{\tau} \int_{|v|>M} \log \left\{\frac{\left(v^{2}-2 j(t) v+h(t)\right)}{\left(v^{2}-2 j(t) v+j^{2}(t)\right)}\right\} d v=O\left(\frac{1}{M} \log n\right) \text {. }
$$




\section{Hence}

(4.14)

$$
\begin{aligned}
& \frac{1}{4 \pi^{2}} \int_{T_{0}}^{n \delta_{0}} \frac{d \tau}{\tau} \int_{-M}^{M} \log \left\{\frac{\left(v^{2}-2 j(t) v+h(t)\right)}{\left(v^{2}-2 j(t) v+j^{2}(t)\right)}\right\} d v \\
& \quad=\frac{1}{4 \pi^{2}} \int_{T_{0}}^{n \delta_{0}} \frac{d \tau}{\tau} \int_{-M}^{M} \log \left\{\frac{\left(v^{2}-2(2 \sigma+1) v+(2 \sigma+1)(2 \sigma+2)\right)}{\left(v^{2}-2(2 \sigma+1) v+(2 \sigma+1)^{2}\right)}\right\} d v+\xi
\end{aligned}
$$

where $|\xi|<\varepsilon \log n$ and $\varepsilon$ is infinitesimally small. Taking $M$ large, we obtain, from (4.11), (4.13) and (4.14), that

$$
E N(1-\delta, 1-T / N)=K^{\prime} \log n+O(\log n),
$$

where

$$
\begin{aligned}
K^{\prime} & =\frac{1}{4 \pi^{2}} \int_{-\infty}^{\infty} \log \left\{1+\frac{2 \sigma+1}{(v-2 \sigma-1)^{2}}\right\} d v \\
& =\frac{1}{4 \pi^{2}} \int_{-\infty}^{\infty} \log \left(1+\frac{2 \sigma+1}{v^{2}}\right) d v=\frac{1}{2 \pi}(2 \sigma+1)^{1 / 2} .
\end{aligned}
$$

Hence from (2.1), (2.5), (2.6), (2.7), (3.1) and (4.15), we obtain

$$
\begin{aligned}
E N(-\infty, \infty)= & 2 E N(0, \infty) \\
= & 2\{E N(0,1-\delta)+E N(1-\delta, 1-T / n) \\
& \quad+E N(1-T / n, 1+T / n) \\
& +E N(1+T / n, 1+\delta)+E N(1+\delta, \infty)\} \\
= & \frac{1}{\pi} \log n\left\{1+(2 \sigma+1)^{1 / 2}\right\}+O\left((\log n)^{1 / 2}\right) \\
= & \frac{1}{\pi}(1+\sqrt{(2 \sigma+1))} \log n .
\end{aligned}
$$

\section{Acknowledgement}

The authors wish to thank the referee for his valuable comments.

\section{References}

B. F. Logan and L. A. Shepp (1968), 'Real zeros of random polynomials II', Proc. London Math. Soc. (3) 18, 306-314.

B. Williamson (1955), The integral calculus, (Scientific Book Company, Patna, India).

H. Cramer (1954), Mathematical methods of statistics, (Princeton University Press). 
K. Farahmand (1986), 'On the average number of real roots of a random algebraic equation', Ann. Probab. 14, 702-709.

M. Das (1969), 'The average number of maxima of a random algebraic curve', Proc. Cambridge Philos. Soc. 65, 741-753.

M. Kac (1943), 'On the average number of real roots of a random algebraic equation', Bull. Amer. Math. Soc. 49, 314-320.

M. Kac (1959), Probability and related topics in physical sciences, (Interscience, New York).

Orissa University of Agriculture and Technology

Bhubaneswar, 751003

Orissa

India
Department of Statistics

Sambalput University

Jyoti Vihar, 768019

Orissa

India 